\title{
EFFECTS OF ROUGHNESS ON DROPLET APPARENT CONTACT ANGLES ON A FIBER
}

\author{
M.M. Amrei ${ }^{1}$, M. Davoudi ${ }^{2}$, G.G. Chase ${ }^{2}$ and H. Vahedi Tafreshi ${ }^{1 *}$ \\ ${ }^{1}$ Department of Mechanical and Nuclear Engineering, \\ Virginia Commonwealth University, Richmond, Virginia 23284-3015 \\ ${ }^{2}$ Department of Chemical and Biomolecular Engineering, \\ The University of Akron, Akron, $\mathrm{OH} 44325$, USA
}

\begin{abstract}
This paper reports on our investigation of the effects of surface roughness on the equilibrium shape and apparent contact angles of a droplet deposited on a fiber. In particular, the shape of a droplet on a roughened fiber is studied via the energy minimization method implemented in the Surface Evolver finite element code. Sinusoidal roughness varying in both the longitudinal and radial directions is considered in the simulations to study the effects of surface roughness on the most stable shape of a droplet on a fiber (corresponding a global minimum energy state). It is found that surface roughness delays droplet shape transition from a symmetric barrel to a clamshell or an asymmetric barrel profile. A phase diagram that includes the effects of fiber roughness on droplet configurations — symmetric barrel, clamshell, and asymmetric barrel—is presented for the first time. It is also found that droplet apparent contact angle tends to decrease on rough fibers. Likewise, roughness tends to increase the force required to detach a droplet from a fiber but the effect diminishes as droplet size increases relative to the size of surface roughness. The results presented in our study have been compared with experimental data or those from prior studies whenever possible, and good agreement has been observed.
\end{abstract}

Keywords: Droplet-fiber interactions; Droplet shape modeling; Surface roughness; Droplet contact angle

\footnotetext{
*Address correspondence to Hooman V. Tafreshi, email: htafreshi@ vcu.edu; tel.: 804-828-9936; fax: 804-827-7030; URL: http://www.people.vcu.edu/ htafreshi/
} 


\section{INTRODUCTION}

Understanding the interactions between a droplet and a fiber is of great importance to many applications. These applications include, but are not limited to, droplet filtration/separation, spray coating, electronic cooling, health and safety, fog harvesting, protective clothing, and medicine [1-6]. A simple manifestation of this effect in nature is the dew formation on spider webs or cactus spines, where life relies on the interactions between a droplet and a fiber in arid climate. Droplet-fiber interactions have been studied in many pioneering studies, and it has been shown that the apparent contact angle (ACA) $\theta_{a p p}$ of a droplet with a fiber can be quite different from the Young-Laplace Contact Angle (YLCA) obtained for a small droplet of the same liquid deposited on a flat surface

made from the same material [1-6]. Depending on fiber diameter, fiber surface energy, droplet volume, and droplet surface tension, two different conformations have been observed for a droplet deposited on a fiber. The first conformation, the barrel shape, tends to occur for larger droplets (relative to fiber), or for when the YLCA is relatively small. The second conformation, the clamshell, is mostly observed with small droplets, or when the YLCA is relatively high. In the former conformation, the droplet wets the fiber symmetrically while in the latter, the fiber is wetted on one side only. There are also droplet-fiber combinations where both of these conformations can be observed [4-11].

Roughness has been shown to affect the wettability of a surface. Wenzel proposed a relationship between YLCA $\theta_{Y L}$ and the ACA of a droplet on a rough flat surface as $\cos \theta_{a p p}=r \cos \theta_{Y L}$ where $r$ is the ratio of the actual to the projected area of the rough surface [12]. However, the measured contact angles may not always match the predictions of this simple equation (see e.g., [13-19]). The knowledge gap is even wider when it comes to droplet contact angle on rough fibers (see e.g., [20-24]), and this has served as the motivation for undertaking the work presented here. 
The remainder of this paper is structured as follows. First, we introduce our rough fiber equation and discuss the numerical modeling approach used to simulate the 3-D shape of a droplet on such a fiber (Sec. 2). We then present a validation study where we compare the predictions of our numerical simulations with the experiment for a few simple configurations in Sec. 3. Our investigations of the effects of surface roughness, fiber diameter, and droplet volume on the shape and ACAs of a droplet deposited are reported in Sec. 4. In this section, we also study the transverse forces required to detach a droplet from a rough fiber for different droplet-fiber configurations. Finally, the conclusions drawn from the work are given in Sec. 5.

\section{NUMERICAL SIMULATIONS}

The surface energy minimization method implemented in the Surface Evolver (SE) finite element code is used to simulate the 3-D shape of a droplet deposited on a rough fiber. SE has shown to be accurate in predicting the airwater interface stability (see e.g., [25-28]). In this section, we first present the equations for producing a fiber having an arbitrary 3-D roughness, and then derive an equation for the energy of a droplet deposited on such a fiber. To our knowledge, no study has yet simulated or quantified roughness on a fiber using a mathematical function. Although real roughness is random in shape and arrangement, we considered sinusoidal roughness for the sake of simplicity (sinusoidal functions have also been used to model roughness on a flat surface [17 and 29]). A rose function (a sinusoid in polar coordinates) can generate sinusoidal roughness at each cross-section of the fiber [30]. By multiplying that equation by another sinusoidal function for the direction along the fiber axis, we obtain a 3-D roughness for a fiber as shown in Fig. 1a.

Consider a fiber in the $x$-direction with a sinusoidal roughness in the axial and preferential directions, described as

$R(x, \alpha)-r_{f}\left[1+a \sin \left(\frac{2 \pi}{\lambda r_{f}} x\right) \sin (\omega \alpha)\right]=0$ 
where $r_{f}$ is the smooth fiber radius, $R(x, \alpha)=\sqrt{y^{2}+z^{2}}$ is the local radius of the rough fiber at any point, and $\alpha=\operatorname{Arctan} \frac{z}{y}$ is angular position. In this equation, $a$ is roughness amplitude, $\lambda$ is roughness wavelength, and $\omega=\frac{2 \pi}{\lambda}$ is the angular frequency of the roughness peaks (see Fig. 1a). For the sake of convenience, we define dimensionless roughness amplitude as $=\frac{a}{r_{f}}$ (note that $b=a$ if $r_{f}=1$ ). SE is used here to obtain the equilibrium 3-D shape of a droplet deposited on a rough fiber by minimizing the total energy of the droplet-fiber system. For a single-droplet-single-fiber system, the total free energy $E$ can be written as

$E=\sigma_{L G} A_{L G}-\sigma_{L G} \int_{A_{S L}} \cos \theta_{Y L} d A+\int \rho h g d V$

where $\sigma_{L G}$ is the surface tension of the liquid and $A_{L G}$ and $A_{S L}$ are liquid-gas and solid-liquid areas, respectively. Here, $h$ represents the vectorial change in the droplet's centroid position caused by body forces (zero in the absence of external forces), $g$ stands for the body force per unit mass, $\rho$ represents the liquid density, and $d A$ and $d V$ are area and volume elements, respectively.

Our simulations start by placing a droplet with an arbitrary shape, but a fixed volume $V$, over the fiber and allowing it to evolve to reach an equilibrium shape and position while maintaining a fixed YLCA at the threephase contact line. The Surface Evolver evolves the droplet shape via a gradient descent method toward a minimal energy. In each iteration the contact line slips smoothly over the bumps in a way that energy of the system decreases until an equilibrium state is reached. A constraint was placed on the fiber surface to prevent any portion of the droplet body to overlap with the solid fiber (as the alternative would be non-physical). Therefore, there would only remain certain areas of a valley between two roughness peaks that a droplet contact line could reside at equilibrium. It is also important to note that solution convergence in these simulations depends more on the mesh density long the contact line than it depends on the total number of mesh used for the simulation. Convergence can often be achieved with a coarse mesh when the fiber is smooth. For a rough fiber however, 
adaptive (dense) mesh near the contact line may become necessary to capture the surface roughness. We used a mesh size equal to $\frac{\lambda}{12}$ along the contact line for rough fibers.

We also calculated the mean curvature of the droplet at each point on the droplet surface (same at all points) for a few cases, and used it in the Laplace equation to obtain the droplet pressure. This pressure was then compared with that calculated by SE for further validation and very good agreement was observed.

The tangent to the inflection point of a droplet profile on a fiber has been considered as the ACA of the droplet in this work (see Fig. 1b). While we initially determined the ACAs by fitting a curve into digitized profiles of each droplet to find the inflection point (where the second derivative of the profile goes to zero), we later realized that similar ACAs can be measured from a computer screen by naked eyes (with an average margin of error of about 3 degrees). The latter was considered in our work for its convenience.

As it has been discussed in the literature, there are infinite number of ACAs (each corresponding to a local minimum energy) that a droplet can exhibit on a rough surface depending on the position of its contact line on the surface asperities [13-19]. Figure 1c shows examples of droplet profiles that can be observed for a droplet with a volume of $V=0.84 \mathrm{~nL}$ and an YLCA of $\theta_{Y L}=30^{\circ}$ on a rough fiber with a radius of $r_{f}=15 \mu \mathrm{m}$ and roughness frequency of $\omega=15$ (under a gravitational force per unit mass of $g_{z}=9.8 \mathrm{~N} / \mathrm{kg}$ ). Figure $1 \mathrm{~d}$ shows droplet energy as a function of ACA for $b=0.1$ and $V=0.84 \mathrm{~nL}$ and $3.37 \mathrm{~nL}$. The ACA corresponding to the global minimum energy is then taken as the ACA $\left(32^{\circ}\right.$ and $42^{\circ}$ for $V=0.84 \mathrm{~nL}$ and $3.37 \mathrm{~nL}$, respectively, for the case shown in the figure). In the remainder of this paper, we only report the ACA corresponding to the droplet's global minimum energy for each droplet-fiber combination. In the absence of a universally accepted method for measuring droplet contact angle on a fiber, we used the so-called inflection point method to read the contact angles from droplet images [3]. 


\section{EXPERIMENT}

A series of experiments has been conducted for validation purposes, and are reported throughout the paper whenever possible. These experiments were conducted using smooth Polypropylene (PP) fibers supplied by Minifibers Inc. Propylene Glycol (PG) and Ultra-Low Sulfur Diesel (ULSD) were used in our experiments. PG was obtained from Fisher scientific, and ULSD was purchased locally. Note that compositions of ULSD vary in time and location based on the source and supplier, and it may have contained some small percentage of biodiesel. Nevertheless, droplet behavior depends on the chemical composition through the surface tension and fluid density, which both were measured before the experiment (see the fluid properties in Table 1 and Figure 2a for a schematic of the experimental setup).

The size of the droplets deposited on the fibers was controlled using the syringe approach [31-32]. In this method, two additional fibers with the same material as the test fiber were used. The first fiber was partially inserted into the needle of the syringe while the second fiber was curled to form a loop and brought into contact with the tip of the needle. When the syringe plunger is pushed, a droplet with a known volume is formed and it is then transferred to the looped fiber. When the droplet is trapped inside the loop, it can then be easily transferred to the test fiber upon contact. While this method allows placing small droplets on a fiber fairly accurately, it is still possible to leave a small droplet at the needle's tip or on the auxiliary fibers. The droplets were imaged using an Olympus DP25 camera (a 5-mega pixel digital color microscope camera). The droplets' volume were estimated by using a micro-syringe in the experiments and also by weighing the droplet. Since none of these methods is accurate enough for very small droplets, we also used an image-based method for axisymmetric droplets. In this method, the digitized image of the droplet is used to produce a mathematical fit to the upper half of its profile. 
This mathematical function is then used to obtain droplet's volume via a simple integration in the axisymmetric domain [33]. Each experiment was repeated three times and the results were averaged.

\section{RESULTS AND DISCUSSION}

In this section, we first investigate the 3-D shape of a droplet deposited on a roughened fiber, and then, we study the forces required to detach the droplet from the fiber in the transverse direction.

\subsection{DROPLET EQUILIBRIUM SHAPE}

Generally speaking, the possible droplet shapes on a fiber are the barrel (symmetric or asymmetric) and clamshell shapes. These configurations have been discussed in the form of phase diagrams in many previous studies for droplets on smooth fibers (see e.g., [8]). In fact, we observed good agreement between the results reported in [8] and those obtained in our preliminary simulations when we considered the same fluid and fiber properties used in [8]. The fibers used in [8] were smooth and fluid properties were quite different from those of our study in this paper and so the comparison is not shown for the sake of brevity. We start this subsection by first presenting a comparison between experimental and computational data obtained for an ULSD or PG droplet shape on a supposedly smooth Polypropylene fiber in terms of its volume for the purpose of examining the accuracy of our numerical simulations. We then move on to produce a phase diagram for droplet shape on a rough fiber.

Figure $2 \mathrm{~b}$ shows an ULSD droplet with a volume of $V=1.35 \mathrm{~nL}$, deposited on a PP smooth fiber next to its computational counterpart. The YLCA for ULSD with a flat PP surface was measured to be $\theta_{Y L}=10^{\circ}$. Figure $2 \mathrm{c}$ compares a real and virtual PG clamshell droplets having a volume ratio of $V=1.54 \mathrm{~nL}$ on a PP fiber with a

YLCA of $\theta_{Y L}=22^{\circ}$. The ACAs measured from the imaged and simulated droplets are presented in the figures, 
and they are in good agreement with one another. Similar comparison is given in Fig. $2 \mathrm{~d}$ for an ULSD droplet with $V=0.215 \mu \mathrm{L}$. The upper $\left(\theta_{a p p}^{u}\right)$ and lower $\left(\theta_{a p p}^{l}\right)$ ACAs are measured from the experimental images and were found to be $41^{\circ}$ and $84^{\circ}$, respectively, which are close to their numerical counterparts of $\theta_{a p p}^{u}=43^{\circ}$ and $\theta_{a p p}^{l}=83^{\circ}$. Note that similar agreement between contact angle values obtained from experiment and numerical simulation has been observed for many other droplet volumes, but not reported here for the sake of brevity.

Increasing the volume of a droplet causes a symmetric barrel-shaped droplet to start becoming asymmetric with respect to the fiber axis due to gravitational effects. To quantify this, we define an asymmetry factor $\varepsilon=h_{1} / h_{2}$ to represent the ratio of the distances between the fiber axis and the uppermost $h_{1}$ and lowermost $h_{2}$ boundaries of the droplet. Figure 3a shows the asymmetry factor versus droplet volume for a ULSD droplet deposited on a PP smooth fiber with a radius of $r_{f}=15 \mu \mathrm{m}$. In this paper, we arbitrarily choose an asymmetry factor of $\varepsilon=0.85$ as the lower limit for a barrel shaped droplet to be referred to as symmetric. As can be seen in Figure 3a, experimental and numerical results are in good general agreement with one another. The slight mismatch between the experimental and numerical results seems to originate from 1 ) the assumption of $\theta_{Y L} \approx 10^{\circ}$ for ULSD with PP surface, and 2) the difficulties in measuring the volume of a clamshell droplet on a fiber accurately. The sudden decrease (from $\varepsilon \approx 0.1$ to $\varepsilon=0$ ) in the data obtained from simulations for a droplet volume of about $V=0.83$ $\mu \mathrm{L}$ indicates that the asymmetric barrel-shaped droplet has transformed to a clamshell droplet.

Figure $3 \mathrm{~b}$ shows the effects of fiber roughness on droplet shape obtained for a fiber with a diameter of $r_{\mathrm{f}}=$ $15 \mu \mathrm{m}$, a roughness frequency of $\omega=15$, an YLCA of $\theta_{\mathrm{YL}}=30^{\circ}$, but different fiber roughness amplitudes of $\mathrm{b}=0,0.01$, and 0.1 . These results indicate that for rougher fibers, the transition from a symmetric barrel to an asymmetric barrel shape takes place at a larger droplet volume. In other words, a barrel shape droplet has a stronger tendency to remain symmetric when the fiber is rougher. The inset of Fig. $3 \mathrm{~b}$ shows the volume of the 
largest droplet that can remain attached to a fiber under gravity. It can be seen that maximum droplet volume increases with increasing fiber roughness. These results are consistent with the predictions of the Wenzel equation: roughness makes a "philic" surface more "philic".

Figure 4 shows a phase diagram obtained numerically for possible configurations of a droplet on a rough fiber. Here, we considered a fiber with a radius of $r_{f}=15 \mu \mathrm{m}$, a YLCA of $\theta_{Y L}=30^{\circ}$, and a roughness frequency of $\omega=15$. In this figure, the squares, triangles, and circles represent the conditions where the possible droplet configurations are symmetric barrel shape, coexisting symmetric barrel and clamshell, and coexisting asymmetric barrel and clamshell droplets, respectively. The symmetric barrel shape seems to be the dominant droplet shape when the droplet volume is small or when the fiber roughness amplitude is high. With increasing the droplet volume or decreasing the roughness amplitude (on a relative basis), the clamshell shape also becomes a possibility. To quantify the degree of asymmetry in barrel-shaped droplets, the asymmetry factor $\varepsilon$ (not applicable to clamshell droplets) is obtained and is added to Fig. 4. It can be seen that $\varepsilon$ increases with increasing fiber roughness b indicating that a droplet can better retain its symmetric barrel shape on a rough fiber. The effects of gravity is negligible when the droplet is small, and so the droplet shape appears like a symmetric barrel. The effect of gravity becomes more significant when the droplet volume is large; gravity tends to pull the droplet downward causing it appear like an asymmetric barrel. Increasing the roughness of a fiber increases the wetted area (solid-liquid contact area) of the fiber. This in turn, increases the fiber's capillary forces (in comparison to the gravitational force) and delays droplet shape transitioning from a symmetric shape to an asymmetric shape.

\subsection{ApPARENT CONTACT ANGLE}

In this subsection, we investigate how droplet ACA on a fiber varies with fiber roughness. Figure 5a shows how varying $b$ from 0.01 to 0.1 affects a droplet's ACA. It can be seen that ACA decreases with increasing the 
amplitude of the roughness for both droplet volumes considered, although the effect seems to be stronger for the smaller droplet. Note also that droplet ACA increases with increasing droplet volume.

Droplets forming a clamshell shape are studied in Fig. 5b. It can be seen that ACA for a clamshell droplet decreases with increasing fiber roughness. Similar to the results given in Fig. 5a, the rate of decrease in ACA is lower for the larger droplet. We believe the appearance of an upward trend for the droplet with a volume of 0.84 $\mathrm{nL}$ in Fig. $5 \mathrm{~b}$ is due mostly to errors associated with ACA measurement. Figure 5b also shows that ACA increases with increasing volume of the droplet on a fiber. Furthermore, comparing the results given in Fig. $5 \mathrm{~b}$ to those in Fig. 5a, one can see that the ACA is higher for clamshell droplets.

Figure 5c presents upper $\left(\theta_{a p p}^{u}\right)$ and lower $\left(\theta_{a p p}^{l}\right)$ ACAs for asymmetric droplets with two different volumes versus fiber roughness amplitude. It can be seen that $\theta_{a p p}^{l}$ (blue symbols) slightly decreases with increasing fiber roughness, as increasing fiber roughness works against droplet shape becoming asymmetric. The effect of roughness on $\theta_{a p p}^{u}$ however, seems to be negligible. Generally speaking, effects of roughness becomes negligible when droplets are larger as was previously discussed for flat surfaces [13-19].

To investigate how surface roughness frequency $\omega$ can affect the ACA of a droplet, we considered a barrel shape droplet with a volume ratio of $V=3.37 \mathrm{~nL}$ in Fig. 6 . This figure shows only the ACA corresponding to the global minimum energy of the droplet as a function of fiber roughness frequency $\omega$. It can be seen that ACA decreases as $\omega$ increases, but the change in ACA tends to become negligible as roughness frequency increases. This observation is consistent with that reported in [13-19] for the effects of frequency of variation of a surface chemical heterogeneity (or droplet volume relative to heterogeneity length scale) on ACA. Our numerical 
simulations indicate that roughness amplitude has generally a greater effect on a droplet ACA than roughness frequency for the range of the dimensions studied in this paper.

\subsection{DETACHMENT FORCE}

Our study in this section is aimed at predicting the force required to detach a droplet from a fiber in the direction normal to the fiber axis. To do this, we first obtain an equilibrium shape for the droplet on the fiber in the absence of any body force. We then apply a small body force to the droplet and incrementally increase it with an arbitrary increment of $\Delta g_{z}=9.8 \mathrm{~N} / \mathrm{kg}$ (one gravity) until no stable shape can be obtained for the droplet on the fiber. The largest body force under which a stable droplet shape could be obtained (plus an increment of $\Delta g_{z}$ ) is then taken as the force required to detach the droplet from the fiber. It should be noted that for the YLCA range considered in our study, it is quite possible for a detaching droplet to break up into a large portion leaving the fiber and a small portion remaining on the fiber (leaving a residue behind on the fiber). Note that the simulation method used in this work cannot be used to model the dynamics of droplet detachment or the volume of the droplet residue on the fiber after detachment.

Figure 7 shows the force required to detach a droplet from a fiber versus droplet volume for different surface roughness amplitudes. The inset in Fig. 7 shows a droplet with a volume of $V=0.84 \mathrm{~nL}$ under different body forces. It can be seen that the droplet's original symmetric barrel shape transforms first to an asymmetric profile and then to a clamshell configuration upon increasing the body force. The droplet eventually detaches from the fiber with an elongated clamshell shape. The results presented in Fig. 7 indicate that detachment force per unit mass decreases with increasing droplet volume. More interestingly, it can be seen that detachment force increases with increasing fiber roughness (roughness appears to make the fibers more philic), but the effect becomes negligible as droplet size increases. 
In a pioneering study, the cantilever tip in an AFM microscope was used to detach a droplet from a smooth fiber and measure the detachment force [36,37]. This unique approach allowed the investigator to examine variety of fluids on "philic" and "phobic" fibers and to produce an empirical correlation for the detachment force of a droplet from a fiber in terms of droplet contact-line length on the fiber and fluid's surface tension (but not droplet size or its contact angle with the fiber) [37]. The correlation in [37] however, over-predicts the detachment forces obtained in our study by 6 orders of magnitude. We conjecture that the mismatch is due to the use of inconsistent units in this correlation (and the data given Figures 6, 7, and 8 of ref. [37]). The detachment forces shown in Figure 7 for the case of the smooth fiber are on the same order of magnitude as those in [34, 35, and 36].

It is worth mentioning that the values reported here for droplet detachment force per mass from a fiber with a radius of $15 \mu \mathrm{m}$ can be used to predict those for droplets with different diameters (or surface tensions and/or densities) from fibers with different diameters as long as the Bond number $\left(B o=\frac{\rho g r^{2}}{\sigma}\right)$ and the dimensionless droplet volume $\frac{V}{r_{f}^{3}}$ are kept constant. For a constant Bond number and a constant dimensionless droplet volume, changing the fiber diameter from a diameter $d_{1}$ to diameter $d_{2}$, increases the detachment force per mass by a factor of $\left(\frac{\sigma_{2}}{\sigma_{1}}\right)\left(\frac{\rho_{1}}{\rho_{2}}\right)\left(\frac{\cos \theta_{Y L 2}}{\cos \theta_{Y L 1}}\right)^{\xi}\left(\frac{d_{1}}{d_{2}}\right)^{2}$ where $\sigma_{1}$ and $\theta_{Y L 1}$, and $\sigma_{2}$ and $\theta_{Y L 2}$ are the surface tension and YLCA of the droplet on the fiber with diameter $d_{1}$, and on the fiber with diameter $d_{2}$, respectively. $\xi$ is number that may vary between zero and one, and it characterizes the impact of the YLCA on detachment force-a parameter that can only be determined accurately in a future investigation. Using such relationships, one can see that the droplet detachment forces reported here for smooth fibers are in good agreement with those reposted previously in [28] for droplets with different diameters and fluid properties deposited on fibers with different diameters. 


\section{CONCLUSIONS}

The wetting behavior of a droplet deposited on a rough fiber is investigated in this paper. The results of our study is condensed into a phase diagram that, unlike those reported previously [8], includes the effects of fiber roughness on droplet configurations. Our results indicate that the occurrence of different droplet shape configurations on a fiber (symmetric barrel, clamshell, and asymmetric barrel) depends on fiber roughness, droplet volume, and fiber radius. In particular, it was shown that roughness increases the tendency of a droplet to retain its symmetric barrel shape as droplet volume is increased. Following the established knowledge for a droplet on a flat surface comprised of 2-D roughness or chemical heterogeneity [15], we quantified the effects of surface roughness on the most stable apparent contact angle (corresponding to a global minimum energy) attainable for a droplet deposited on a fiber with 3-D roughness. It was found that apparent contact angle decreases with fiber roughness, however the effect becomes less significant when increasing the droplet size relative to roughness amplitude or frequency $[15,18]$. We also calculated the force required to detach a droplet from a rough fiber, and showed that this force increases with increasing fiber roughness or decreasing droplet volume. Likewise, the effects of roughness of detachment force becomes less significant when droplet volume was increased.

\section{ACKNOWLEDGMENTS}

The authors acknowledge the support of the National Science Foundation CBET program [1402336 and 1402655]. 


\section{REFERENCES}

1. B.J. Carroll, Equilibrium conformation of liquid drops on thin cylinders under forces of capillarity, J. Colloid Interface Sci. 57 (1976) 488-495.

2. B.J. Carroll, The equilibrium of liquid-drops on smooth and rough circular-cylinders, J. Colloid Interface Sci. 97 (1984) 195-200.

3. S. Rebouillat, B. Letellier, B. Steffenino, Wettability of single fibers-beyond the contact angle approach, Adhes. Adhes. 19 (1999) 303-314.

4. G. McHale, M.I. Newton, B.J. Carroll, The Shape and stability of small liquid drops on fibers. Oil Gas Sci. Technol. 56 (2001) 47-54.

5. G. McHale, M.I. Newton, Global geometry and equilibrium shapes of liquid drops on fibers, Colloid. Surface. A. 206 (2002) 79-86.

6. A. Kumar, Measurement of contact angles from the shape of the drop on a vertical fiber, J. Colloid Interface Sci. 136 (1990) 455-469.

7. E. Lorenceaua, C. Clanetb, D. Quéré, Capturing drops with a thin fiber, J. Colloid Interface Sci. 279 (2004) 192-197.

8. T.-H. Chou, S.-J. Hong, Y.-E. Liang, H.-K. Tsao, Y.-J. Sheng, Equilibrium phase diagram of drop-onfiber: coexistent states and gravity effect, Langmuir 27 (2011) 3685-3692.

9. H.B. Eral, J. de Ruiter, R. de Ruiter, J.M. Oh, C. Semprebon, M. Brinkmann, F. Mugele, Drops on functional fibers: from barrels to clamshells and back, Soft Matter 7 (2011) 5138-5143.

10. R. de Ruiter, J. de Ruiter, H.B. Eral, C. Semprebon, M. Brinkmann, F. Mugele, Buoyant droplets on functional fibers, Langmuir 28 (2012) 13300-13306.

11. M. Mei, J. Fan, D. Shou, The gravitational effect on the geometric profiles of droplets on horizontal fibers, Soft Matter 9 (2013) 10324-10334.

12. R.N. Wenzel, Resistance of solid surfaces to wetting by water, Ind. Eng. Chem. 28 (1936) 988-994. 
13. G. Wolansky, A. Marmur, Apparent contact angles on rough surfaces: the Wenzel equation revisited, Colloid. Surface. A. 156 (1999) 381-388.

14. G. McHale, Cassie and Wenzel: Were they really so wrong?, Langmuir 23 (2007) 8200-8205.

15. A. Marmur, E. Bittoun, When Wenzel and Cassie are right: Reconciling and global considerations, Langmuir 25 (2009) 1277-1281.

16. F.J. Montes Ruiz-Cabello, M.A. Rodríguez-Valverde, A. Marmur, M.A. Cabrerizo-Vílchez, Comparison of sessile drop and captive bubble methods on rough homogeneous surfaces: A numerical study, Langmuir 27 (2011) 9638-9643.

17. R.G. Cox, The spreading of a liquid on a rough solid surface, J. Fluid Mech. 131 (1983) 1-26.

18. R. David, A.W. Neumann, Contact angle hysteresis on randomly rough surfaces: a computational study, Langmuir 29 (2013) 4551-4558.

19. D. Quéré, Wetting and roughness, Annu. Rev. Mater. Res. 38 (2008) 71-99.

20. B.J. Carroll, The equilibrium of liquid drops on smooth and rough circular cylinders, J. Colloid Interface Sci. 97 (1984) 195-200.

21. Y. Zheng, H. Bai, Z. Huang, X. Tian, F. Nie, Y. Zhao, J. Zhai, L. Jiang, Directional water collection on wetted spider silk, Nature, 463 (2010) 640-643.

22. J.K. Gac, L. Gradon, Modeling of axial motion of small droplets deposited on smooth and rough fiber surfaces, Colloid. Surface. A. 414 (2012) 259-266.

23. Y. Chen, Y. Zheng, Bioinspired micro-/nanostructure fibers with a water collecting property, Nanoscale 6 (2014) 7703-7714.

24. C.S. Funk, B. Winzer, W. Peukert, Correlation between shape, evaporation mode and mobility of small water droplets on nanorough fibers, J. Colloid Interface Sci. 417 (2014) 171-179.

25. M.M. Amrei, H.V. Tafreshi, Effects of hydrostatic pressure on wetted area of submerged superhydrophobic granular coatings. Part II: poly-dispersed coatings, Colloid. Surface. A. 481 (2015) $547-560$. 
26. T.M. Bucher, M.M. Amrei, H.V. Tafreshi, Wetting resistance of heterogeneous superhydrophobic coatings with orthogonally layered fibers, Surf. Coat. Tech. 277 (2015) 117-127.

27. H. Aziz, M.M. Amrei, A. Dotivala, C. Tang, H.V. Tafreshi, Modeling Cassie droplets on superhydrophobic coatings with orthogonal fibrous structures, Colloids and Surfaces A. 512 (2017) 6170.

28. M.M. Amrei, D.G. Venkateshan, N. D’Souza, J. Atulasimha, and H.V. Tafreshi, Novel Approach to Measuring the Droplet Detachment Force from Fibers, Langmuir 2016, 32, 13333-13339.

29. A. Promraksa and L.-J. Chen, Modeling contact angle hysteresis of a liquid droplet sitting on a cosine wave-like pattern surface. J. Colloid Interface Sci. (2012), 384, 172-181.

30. H. Martyn Cundy and A.P. Rollett, Mathematical Models, $2^{\text {nd }}$ ed., (1961) (Oxford University Press), p. 73.

31. J. Fang, M. Davoudi, G.G. Chase, Drop movement along a fiber axis due to pressure driven air flow, Sep. Purif. Technol. 140 (2015) 77-83.

32. M. Davoudi, J. Fang, G.G. Chase, Barrel shaped droplet movement at junctions of perpendicular fibers with different orientations to the air flow direction, Sep. Purif. Technol. 162 (2016) 1-5.

33. S.F. Chini, A. Amirfazli, A method for measuring contact angle of asymmetric and symmetric drops, Colloid. Surface. A. 388 (2011) 29-37.

34. R.P. Sahu, S. Sinha-Ray, A.L. Yarin, B. Pourdeyhimi, Blowing drops off a filament, Soft Matter 9 (2013) $6053-6071$.

35. R. Dufour, A. Dibao-Dina, M. Harnois, X. Tao, C. Dufour, R. Boukherroub, V. Senez, V. Thomy, Electrowetting on functional fibers, Soft Matter 9 (2013) 492-497.

36. B.J. Mullins, A. Pfrang, R.D. Braddock, T. Schimmel, G. Kasper, Detachment of liquid droplets from fibres-Experimental and theoretical evaluation of detachment force due to interfacial tension effects. J. Colloid Interface Sci. (2007) 312, 333-340.

37. C.J. Hotz, R. Mead-Hunter, T. Becker, A.J.C. King, S. Wurster, G. Kasper, B.J. Mullins, Detachment of droplets from cylinders in flow using an experimental analogue. J. Fluid Mech. (2015) 771, 327-340. 


\section{ABBREVIATIONS:}

ACA: Apparent Contact Angle

PP: Polypropylene

PG: Propylene Glycol

SE: Surface Evolver

ULSD: Ultra Low Sulfur Diesel

YLCA: Young-Laplace Contact Angle 


\section{FIGURE CAPTIONS}

Fig. 1: Side and cross-sectional views of our virtual rough fiber is shown in (a). The inflection point and apparent contact angle are shown in (b). Overlaid images of droplet profiles corresponding to different local minimum energies are shown in (c) for a droplet with a volume of $V=0.84 \mathrm{~nL}$ on a rough fiber with $r_{f}=15 \mu \mathrm{m}, \theta_{Y L}=$ $30^{\circ}$ and $\omega=15$. Droplet surface energy is plotted versus apparent contact angle in (d) for droplet volumes of $V=0.84 \mathrm{~nL}$ (black symbols) and $V=3.37 \mathrm{~nL}$ (blue symbols). This figure is intended for color reproduction on the web.

Fig. 2: A schematic of the experimental setup is shown in (a) along with an SEM image of the polypropylene (PP) fiber used in the experiments (30 $\mu \mathrm{m}$ in diameter). One-on-one comparison between droplet shape and apparent contact angles on a smooth fiber with $r_{f}=15 \mu \mathrm{m}$ obtained from experiment and numerical simulation for an ULSD droplet with $V=1.35 \mathrm{~nL}$ and $\theta_{Y L} \simeq 10^{\circ}$ in (b), a PG droplet with $V=1.54 \mathrm{~nL}$ and $\theta_{Y L}=22^{\circ}$ in (c), and an ULSD droplet with $V=0.215 \mu \mathrm{L}$ and $\theta_{Y L} \simeq 10^{\circ} \mathrm{in}(\mathrm{d})$.

Fig. 3: Asymmetry factors from experiment and numerical simulation are shown versus droplet volume in (a) for ULSD droplets on a smooth PP fiber $\left(\theta_{Y L} \simeq 10^{\circ}, r_{f}=15 \mu \mathrm{m}\right)$. Asymmetry factor is shown in (b) for droplets on rough fibers with a radius of $r_{f}=15 \mu \mathrm{m}$ and an YLCA of $\theta_{Y L}=30^{\circ}$ but three different roughness amplitudes of $b=0,0.01$, and 0.10 . The inset figure shows the maximum droplet volume attainable on the same fibers but with different roughness amplitudes.

Fig. 4: A phase diagram showing different possible conformations for a droplet on a rough fiber. Square, delta, and circle represent symmetric barrel drop, coexistence of symmetric barrel and clamshell droplets, and 
coexistence of asymmetric barrel and clamshell droplets, respectively. Here, $r_{f}=15 \mu \mathrm{m}, \omega=15$ and $\theta_{Y L}=30^{\circ}$. The asymmetry factor is given next to some of the symbols for comparison.

Fig. 5: Apparent contact angle is shown versus fiber roughness amplitude for examples of symmetric barrel shaped droplets in (a), clamshell droplets in (b), and asymmetric barrel shaped droplets in (c). For the clamshell droplets both upper (black hollow symbols) and lower (blue filled symbols) apparent contact angles are reported. Here, $r_{f}=15 \mu \mathrm{m}, \omega=15$, and $\theta_{Y L}=30^{\circ}$. This figure is intended for color reproduction on the Web and in print.

Fig. 6: Effects of roughness frequency on apparent contact angle is shown using a barrel shaped droplet with a volume of $V=3.37 \mathrm{~nL}$ on a rough fiber with a radius of $r_{f}=15 \mu \mathrm{m}$ and a YLCA of $\theta_{Y L}=30^{\circ}$.

Fig. 7: The force per unit mass required to detach droplets with different volumes from a rough fiber with a radius of $r_{f}=15 \mu \mathrm{m}$ and a roughness frequency of $\omega=15$, but different roughness amplitudes ranging from $b=0$ to $b=0.1$. The inset shows the equilibrium shape under an increasing external body force perpendicular to the fiber axis for a droplet with a volume ratio of $\frac{V}{r_{f}^{3}}=250$ on a fiber with $r_{f}=15 \mu \mathrm{m}$ and $b=0.1$.

Table1: Physical properties of test liquids at 20 Celsius. 

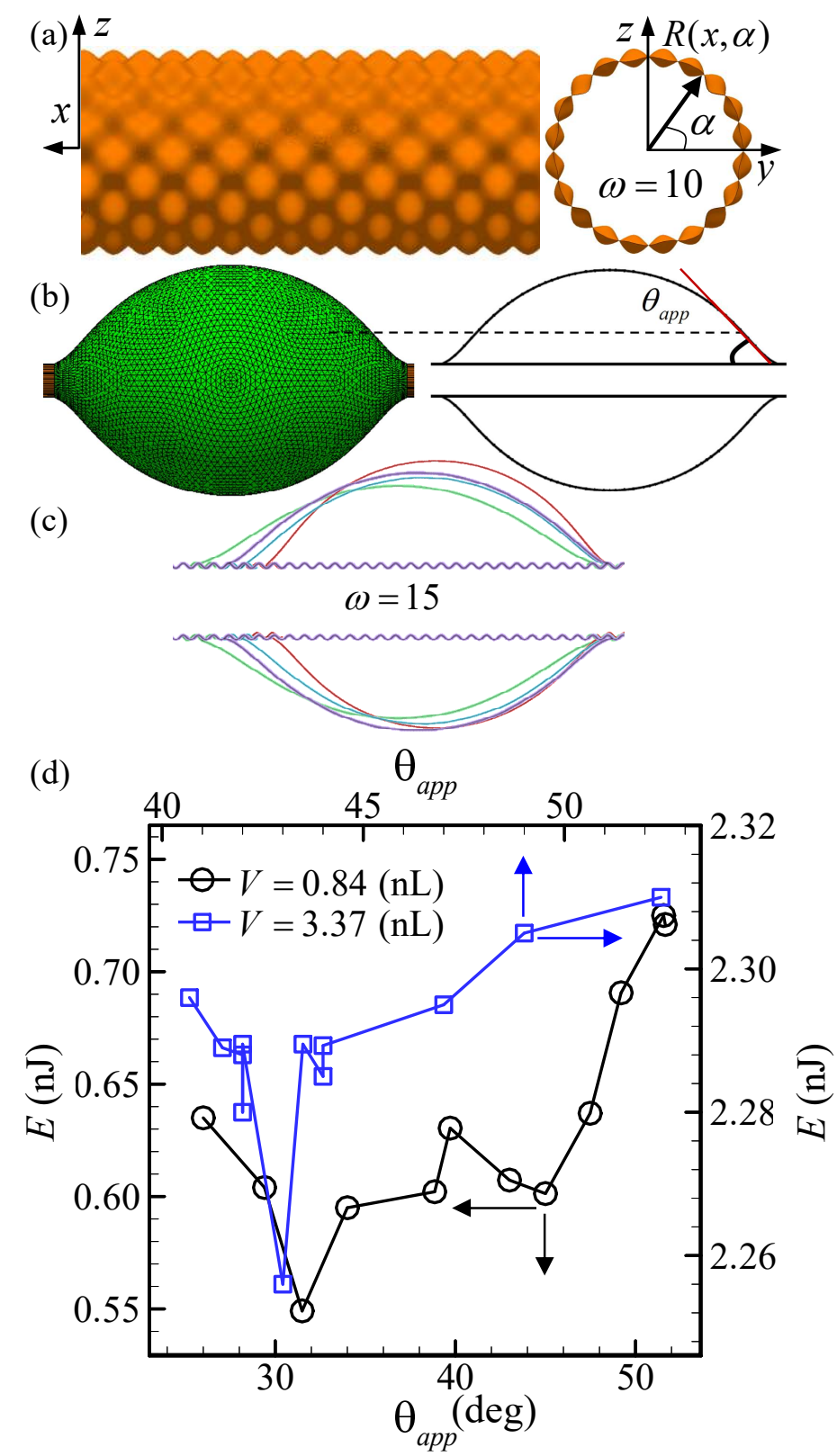

Figure 1: 


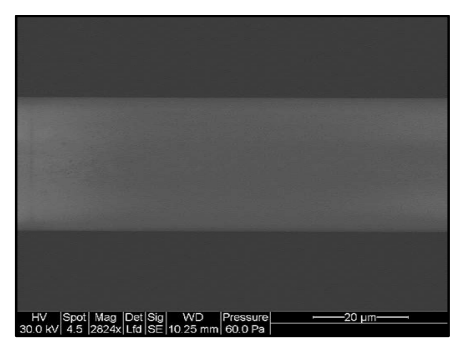

(a)

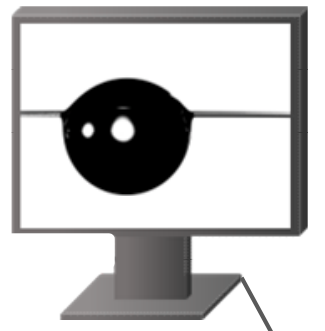

Light

Source

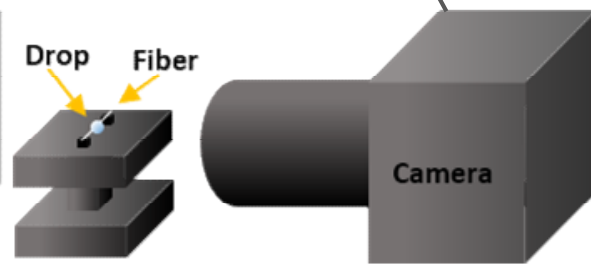

(b)

$$
\theta_{a p p}=42^{\circ}
$$

$\theta_{a p p}=40^{\circ}$

(c)

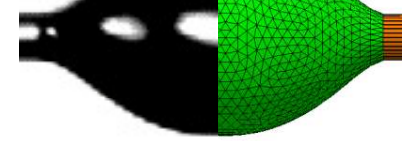

$$
\theta_{a p p}=40^{\circ} \longrightarrow \theta_{a p p}=44^{\circ}
$$

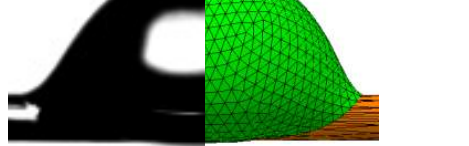

(d)

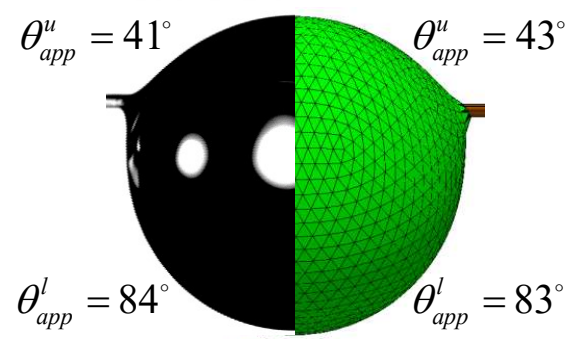

Figure 2: 

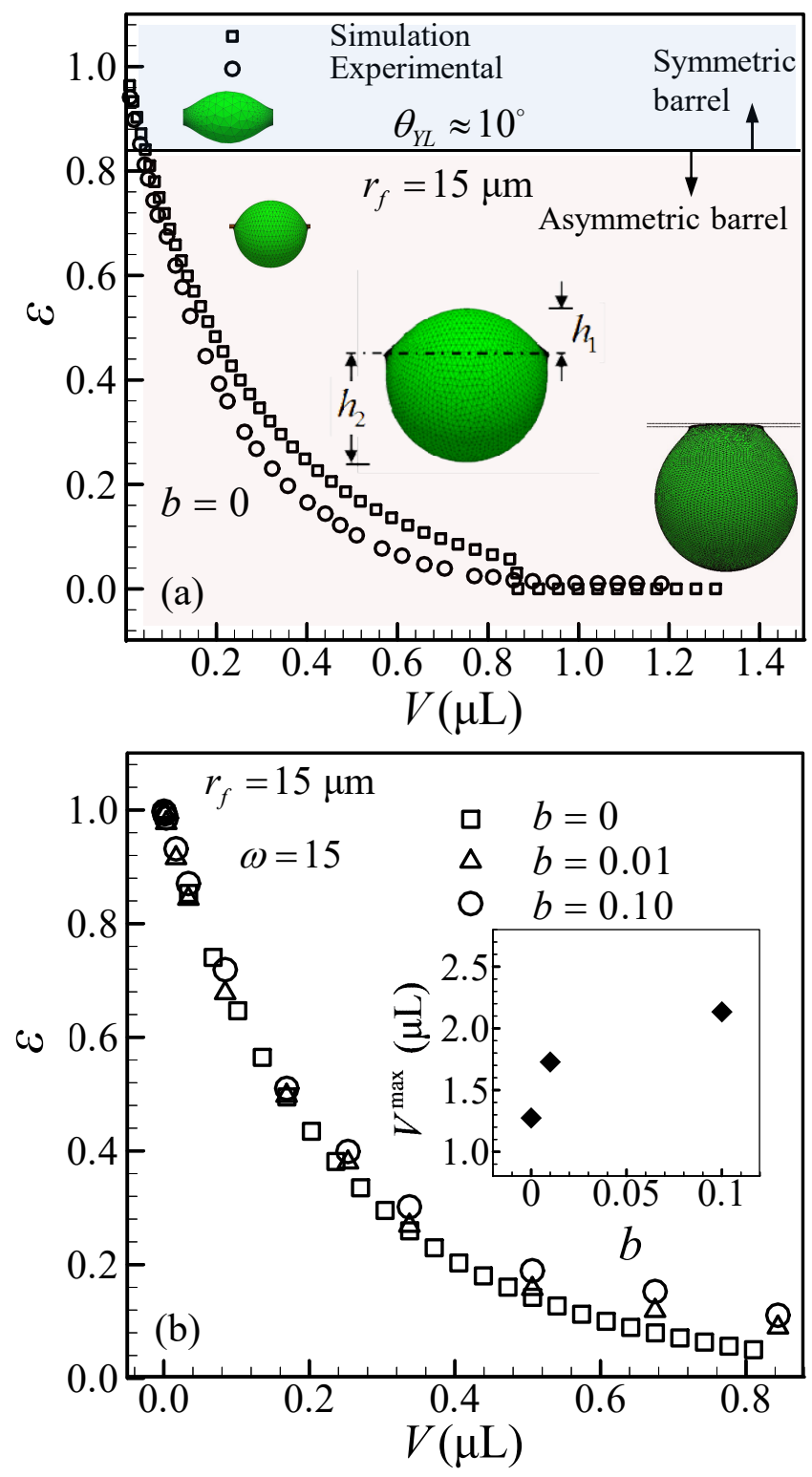

Figure 3: 


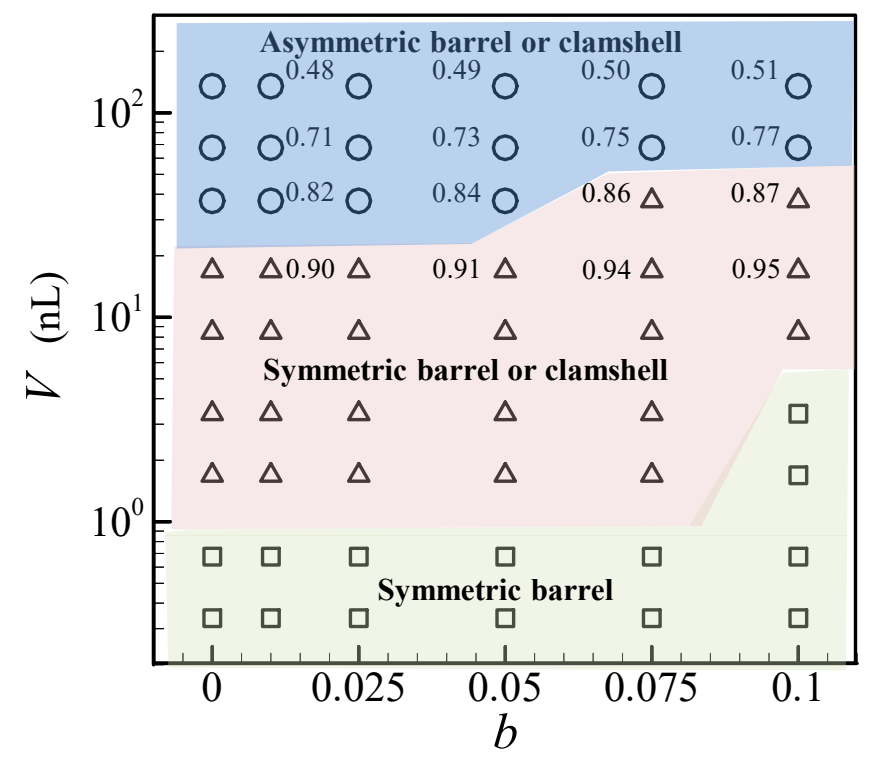

Figure 4: 

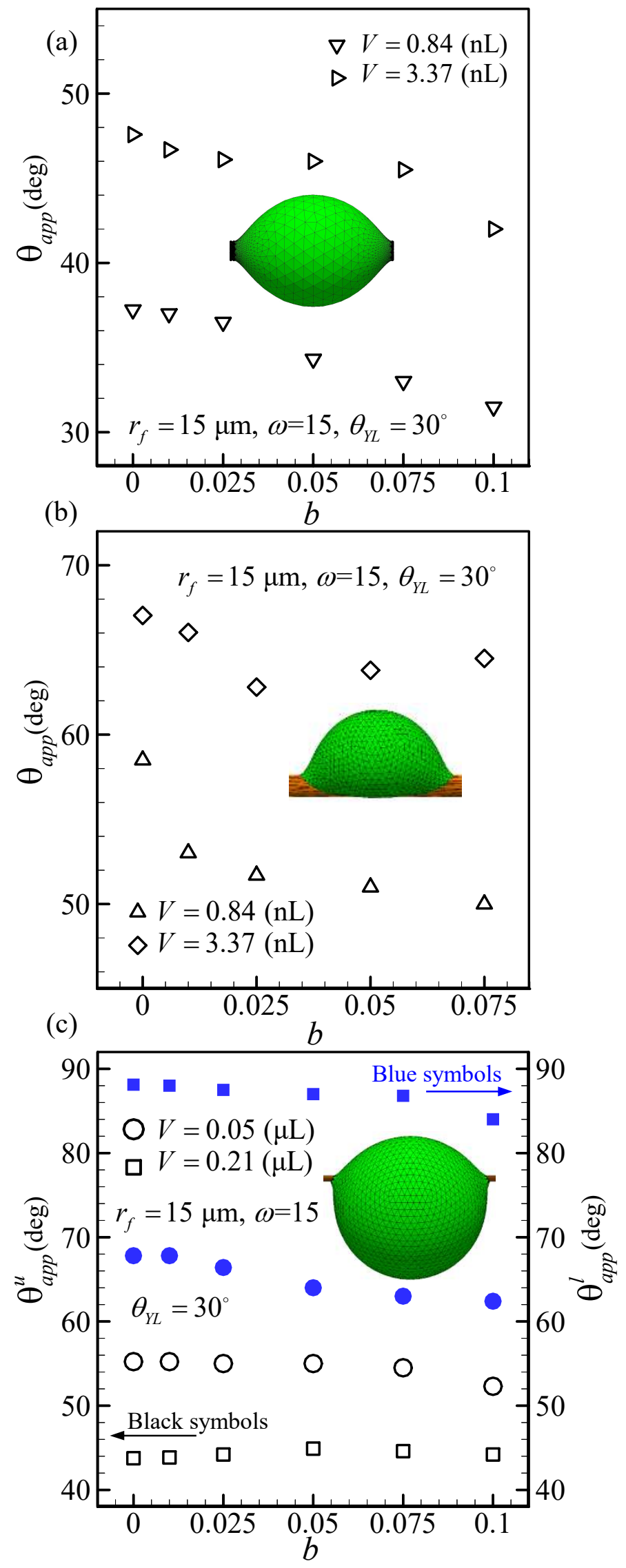

Figure 5: 


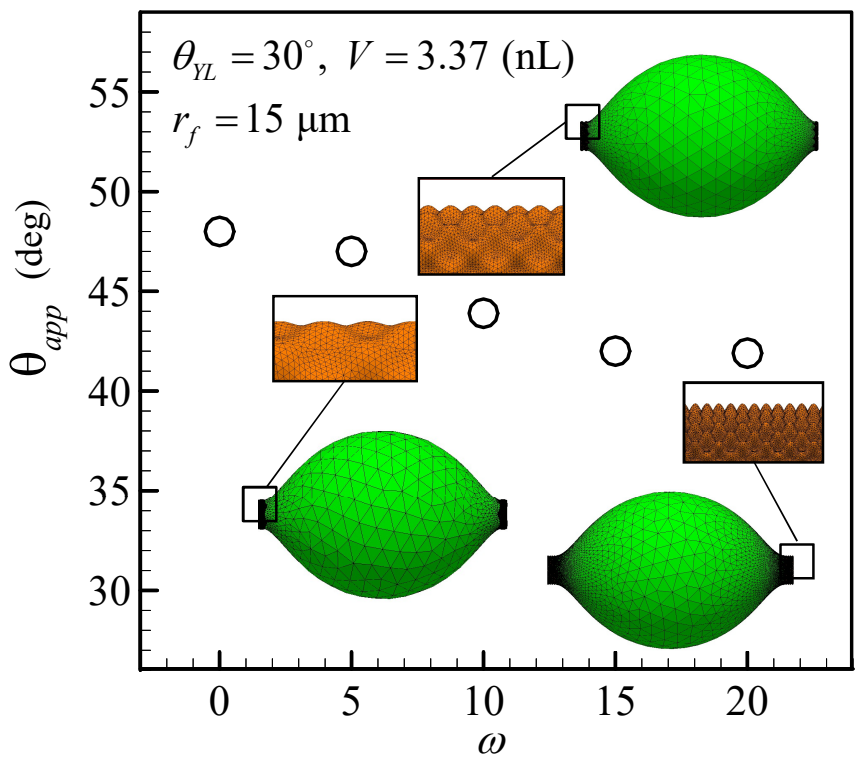

Figure 6: 


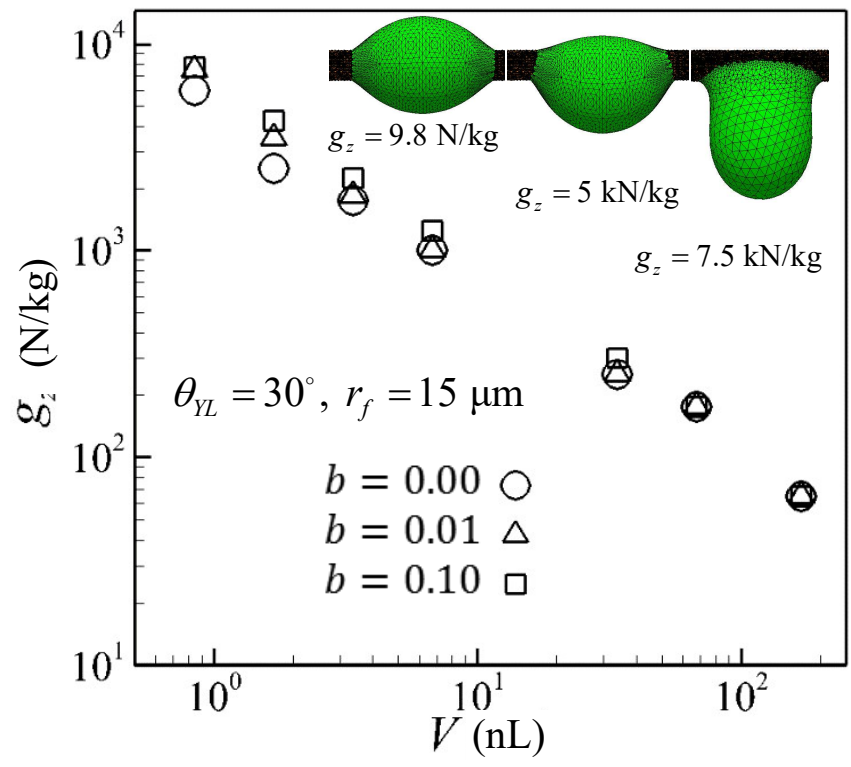

Figure 7: 


\begin{tabular}{|l|c|c|c|}
\hline Liquid & $\begin{array}{c}\text { Surface Tension } \\
(\mathrm{mN} / \mathrm{m})\end{array}$ & Density $(\mathrm{Kg} / \mathrm{m} 3)$ & YLCA (deg.) \\
\hline $\begin{array}{l}\text { Ultra-Low Sulfur Diesel } \\
\text { (ULSD) }\end{array}$ & $\mathbf{2 8}$ & $\mathbf{8 3 0}$ & $\mathbf{1 0}$ \\
\hline Propylene Glycol & $\mathbf{3 2 . 5}$ & $\mathbf{9 8 0}$ & $\mathbf{2 2}$ \\
\hline
\end{tabular}


GRAPHICAL ABSTRACT

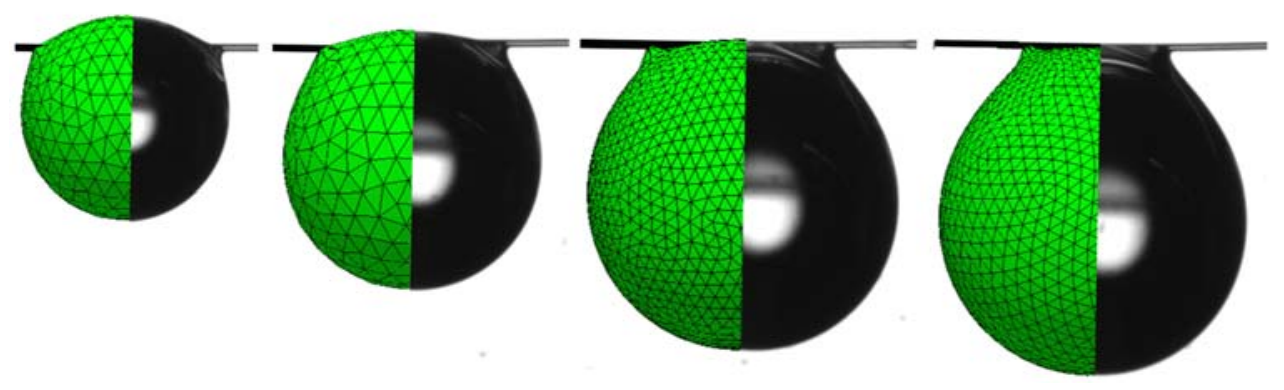

